\title{
A precise form of divisive suppression supports population coding in primary visual cortex
}

\author{
Sean P. MacEvoy, Thomas R. Tucker, and David Fitzpatrick \\ Department of Neurobiology Duke University Medical Center Durham, NC 27710
}

\begin{abstract}
The responses of neurons in the primary visual cortex (V1) to an optimally-oriented grating are suppressed when a non-optimal grating is superimposed. Although cross-orientation suppression is thought to reflect mechanisms that maintain a distributed code for orientation, the impact of superimposed gratings upon V1 populations is unknown. Using intrinsic signal optical imaging, we find that patterns of tree shrew V1 activity evoked by superimposed equal-contrast gratings were predicted by the mean of patterns evoked by individual component gratings. This prediction held across contrasts, for summed sinusoidal gratings or non-summing square-wave gratings, and was reflected in single-unit extracellular recordings. Intracellular recordings revealed consistent levels of suppression throughout the evolution of subthreshold responses. These results indicate that divisive suppression powerfully governs population responses to multiple orientations. Moreover, the specific form of suppression we observe appears to support independent population codes for stimulus orientation and strength, and calls for a reassessment of mechanisms that underlie cross-orientation suppression.
\end{abstract}

The response of a single neuron in the primary visual cortex (V1) to an optimally-oriented grating can be dramatically reduced by the superimposition of an orthogonal grating that does not, by itself, evoke a response 1-3. This "cross-orientation suppression" is generally considered a manifestation of a pervasive system of divisive suppression in V1, contributing to functions such as contrast gain control and redundancy reduction in the coding of natural images 4-10. Although cross-orientation suppression has been well characterized at the level of single neurons, how divisive suppression contributes to population coding in V1 is less clear. Because a single grating activates a broad population of cortical neurons, the absolute level of activity of any particular member of the population provides little information about the orientation of the stimulus. However, the relative activity of neurons in the population provides a robust signal that faithfully represents the orientation of a single grating stimulus and more generally, the 2-D Fourier orientation composition of any stimulus 11-15. In principle, divisive suppression can act to preserve the relative levels of activity among the members of the population, preventing phenomena such as response saturation from distorting the distributed representation $5,16,17$. In this context, cross-orientation

Users may view, print, copy, and download text and data-mine the content in such documents, for the purposes of academic research, subject always to the full Conditions of use:http://www.nature.com/authors/editorial_policies/license.html\#terms

Address for correspondence: Sean MacEvoy Center for Cognitive Neuroscience University of Pennsylvania 3720 Walnut Street Philadelphia, PA 19104 macevoy@psych.upenn.edu. 
suppression can be viewed as a rescaling of the entire population response to maintain an accurate distributed representation of the orientations in the compound grating stimulus.

This view makes the simple prediction that the population response pattern in V1 to superimposed gratings should approximate the scaled linear sum of the responses to the component gratings presented by themselves. While this prediction is straightforward, the available evidence leaves its accuracy uncertain. Early stages of cortical processing are wellcharacterized as linear transformations of input from the lateral geniculate nucleus (LGN), but subsequent stages introduce prominent non-linearities in single cell responses (e.g., center-surround interactions) whose combined impact on the shape of the population tuning function is difficult to predict from single unit recordings18-22. Moreover, available single unit data on the response of single neurons to superimposed gratings is largely limited to descriptions of the impact of a non-optimal grating on the responses of neurons to their preferred grating 1-3, 7, 23. Understanding the impact of a superimposed grating on population activity requires measuring neuronal responses to all possible combinations of two orientations, irrespective of their relationship to each neuron's preferred orientation.

A further complication in understanding the impact of divisive suppression in population coding is the fact that virtually all previous studies of cross-orientation suppression used a single class of stimuli: drifting sinusoidal gratings whose luminance profiles sum when two gratings are superimposed2, 3, 7, 23-27. The merits of using this type of stimulus configuration are substantial28, but it is not known whether the properties of suppression that have been described with this stimulus set can be generalized to superimposed contours whose luminance profiles do not sum linearly. This is a situation that arises frequently in natural scenes (e.g., due to occlusion), and presents no less a challenge to a population code for orientation. In spite of this, two recent studies26, 27 have suggested that crossorientation suppression may arise under stimulus conditions that are unique to additive superimposition of sinusoidal gratings. This would appear to limit the role of divisive normalization in supporting population coding of natural scenes.

The aim of this study was to provide a direct test of the impact of divisive suppression on the population response of V1 neurons. Using intrinsic signal imaging techniques in V1 of the tree shrew, we find that patterns of population activity evoked by superimposed gratings were predicted with high precision by a specific form of divisive rescaling in which responses to equal-contrast grating pairs were the mean of the patterns evoked by each component grating presented alone. Moreover, this outcome was unchanged whether stimuli were composed of summed sinusoids or non-summing square-wave gratings, and held for both high and low stimulus contrasts. Our results not only indicate that divisive suppression plays a fundamental and consistent role in constructing a faithful population representation of multiple simultaneous orientations, but also call for a reassessment of the factors that account for the magnitude of suppression, and the mechanisms that have been proposed to explain it. 


\section{Results}

\section{Population response to superimposed gratings}

We first sought to characterize patterns of population activity evoked by non-summing superimposed gratings differing in orientation. To do so we acquired intrinsic signal optical images of tree shrew V1 during presentation of stationary rectangle-wave gratings presented alone or as superimposed pairs (insets, Fig. 1a-c, f-h). Unless otherwise specified, component gratings always had equal contrasts of 0.5 . Single gratings at $135^{\circ}$ and $45^{\circ}$ (Figures 1A,B) evoked complementary patterns of relatively active (dark) and inactive (light) regions, corresponding to the distribution of preferred orientation across the cortical surface. When the gratings were superimposed, these patterns were almost completely absent (Fig. 1c-e), consistent with the prediction that cortical regions responding vigorously to one grating exhibited a much weaker response when an orthogonal grating was superimposed.

To fully characterize the population response to superimposed gratings, we constructed population response profiles (PRPs) representing the distribution of cortical activity evoked by a stimulus across cortical sites preferring the full range of orientation preferences (Fig. 1f-j; see Experimental Procedures). The distributions of population activity evoked by single gratings at $70^{\circ}$ and $110^{\circ}$ peak very close to $70^{\circ}$ and $110^{\circ}$, confirming the accuracy of the PRP sampling procedure. In contrast, the distribution of population activity evoked by the superimposed grating condition peaks at an intermediate orientation $\left(\sim 90^{\circ}\right)$, and exhibits markedly reduced height compared to the more effective single grating among populations of all orientation preferences.

Broadly consistent with divisive suppression, PRPs for superimposed gratings closely resembled the mean of PRPs for component gratings (Fig. 1j). The component mean predicted responses to the superimposed pair regardless of the preferred orientation of a cortical site, or of the relative responses evoked by the two components. Changing the offset angle between gratings from $90^{\circ}$ to $20^{\circ}$ impacted the shape of the PRP for the superimposed pair, narrowing it and eventually yielding a unimodal distribution (Fig. 2a-c). Nevertheless, for each angle offset PRPs for superimposed gratings closely matched their mean component PRP. Component means also predicted responses to superimposed gratings across a range of spatial frequencies (Supplementary Figure 1).

To quantify this phenomenon, we compared responses to component and superimposed gratings pooled across PRPs from all imaging sessions. Responses to superimposed gratings obeyed a strict linear relationship to their component sums (Fig. 2d). The slope of the bestfit line relating superimposed responses to single-grating responses was $0.51\left(R^{2}=0.83\right)$; perfect prediction of responses to superimposed gratings by their component means would produce a slope of exactly 0.5 . Regressions within individual imaging sessions yielded similar results (Fig. 2e). Based on this analysis, we conclude that the mean component response accurately predicted responses to superimposed gratings across all imaging sessions. 
These results indicate that divisive suppression tightly controls population responses to nonsumming superimposed gratings. This result appears to be inconsistent with recent models suggesting that cross-orientation suppression results from LGN saturation occurring only under conditions generated by drifting summed sinusoidal gratings 26,27 . However, it is possible that the suppression we observed with stationary non-additive gratings was different from suppression that results from summed superimposed gratings. To address this possibility, we repeated our experiment in three animals using drifting sinusoidal gratings. As with non-summing gratings, PRPs for summed sinusoids were well predicted by the mean of their component PRPs (Fig. 3a,b). The ability of the component mean to accurately predict responses to multiple orientations for both additive and non-additive stimuli suggests that cross-orientation suppression is not limited to a particular stimulus configuration, but reflects a more generalized mechanism that regulates overall levels of population activity induced by stimuli that contain multiple orientations.

Models of cross-orientation suppression based on LGN saturation also predict that suppression should be absent when both gratings in a superimposed pair have low contrasts, below the point at which their summed contrasts would produce LGN saturation. Against this prediction, we find that the component mean predicts the population response to superimposed gratings as well at low contrast (Fig. 3c) as it does at high contrast. This was the case even though single-grating responses roughly doubled when contrast was doubled (compare PRP heights for single gratings in Fig. 3a,c), indicating that the summed contrast of the low-contrast gratings was insufficient to saturate the responses of cortical neurons and, by extension, their LGN inputs. Combined data from three animals shows essentially the same relationship between superimposed responses and component responses for high contrast and low contrast gratings (Fig. 3b and d, respectively).

While the component mean accurately predicted responses to superimposed gratings when both components had the same contrast, simple logic dictates that the same prediction could not hold for components with dissimilar contrasts. At the extreme, as the contrast of one grating goes towards zero, the PRP for a superimposed pair should approach that of the remaining grating. Consistent with this prediction, when individual gratings had different contrasts, the PRP for the superimposed pair departed from the component mean and began to resemble that of the higher contrast grating (Fig. 3e,f).

Single-unit studies have shown that cross-orientation suppression is an essentially local phenomenon3. Consistent with this, we found that the component mean predicted population responses to superimposed gratings only at cortical locations with RFs encompassing both components. Activity evoked by an " $\mathrm{X}$ " consisting of a single line at each orientation was well-predicted by the component mean in a region of cortex extending roughly $1 \mathrm{~mm}$ from the representation of the point of crossing between the two lines (Supplementary Figure 2). In the tree shrew, this corresponds to roughly $5^{\circ}$ of visual angle 29 , which is about the size of the typical V1 receptive field in layer $2 / 3$ at this eccentricity30. Regions farther away from the crossing point had responses close to each line presented alone.

In sum, our results show that divisive suppression is a ubiquitous feature of V1 processing that operates on a broader range of stimulus configurations than previously thought. We 
observed essentially identical rescaling of the population response regardless of whether stimuli were summed drifting sinusoids or non-summing stationary rectangle-wave gratings, or whether they appeared at high or low contrast. Further, we find that divisive suppression takes a particular form in which population responses to any two equal-contrast gratings are well-predicted by the mean of the component responses. The remainder of the Results section presents single-unit data which we collected in order to validate optical imaging data, as well as to better understand the nature of the suppression we observed.

\section{Validation of intrinsic signal results}

Although our imaging results indicated that neuronal responses to superimposed gratings were predicted by the mean of their component responses, we wished to ensure that this outcome reflected neuronal activity, and not non-neuronal hemodynamic factors or our imaging techniques. To validate our imaging results, we made single-unit extracellular recordings in layer $2 / 3$ using the same stationary square-wave gratings as used for imaging. Neuronal responses to superimposed gratings were well predicted by the mean of responses to each component presented alone (Fig. 4a-e), indicating that our intrinsic signal images faithfully represented the average activity of V1 neurons to superimposed gratings.

To assess how reliably the component mean predicted responses to paired gratings among individual cells, we computed a suppression index (SI) for each neuron by subtracting from 1 the ratio of the superimposed response to the sum of component responses for each grating pair, and then averaging the resulting values across all 28 unique stimulus pairs tested with each cell (see Experimental Procedures). Under this measure, a cell with responses to superimposed gratings that were the same as the sum of component responses would have an SI value of 0 , a cell exhibiting complete suppression would have an SI of 1, and a cell with superimposed responses exactly equal to component means would have an SI of 0.5. All but two of 15 neurons recorded from five animals had SI values falling in a single cluster between 0.3 and 0.6 (Fig. 4f), further suggesting that our imaging results reflect the behavior of a majority of individual layer $2 / 3$ neurons.

\section{Time course of responses to superimposed gratings}

Our imaging and extracellular experiments demonstrate the consistent nature of the divisive suppression that occurs with superimposed gratings. However, both of these measures are averages of neuronal responses during the stimulus period. It is possible that neuronal responses to superimposed gratings could exhibit complex dynamics that lead them to depart significantly from the component mean, but which are obscured by temporal averaging. For instance, if suppression were instantiated - at least in part - by feedback mechanisms, we would expect to see a delay in suppression relative to response onset. In order to better understand the temporal dynamics of suppression, we made in vivo intracellular recordings from layer $2 / 3$ neurons.

We obtained stable recordings from 15 neurons in 8 animals for a time sufficient ( 1 hour) to collect responses to at least 15 trials of each of the 36 stimuli in our battery. Stimulus and timing parameters were identical to those used during extracellular recordings. Similar to our imaging and extracellular results, responses to paired gratings resembled the mean of 
their component responses consistently among our sample of neurons, and for all grating combinations (Fig. 5a-h). Within the same neuron, membrane potential and firing rates evoked by superimposed gratings were predicted equally well predicted by component means (Supplementary Figure 3).

To more precisely assess how well this prediction held up over time, we repeated the regression analysis used for imaging and extacellular records, comparing each response to a superimposed pair to the sum of responses evoked by its respective components (Fig. 6a-d). Regression slopes for average membrane potential during the first 200, 20, 10, and 5 milliseconds following response onset were $0.48,0.49,0.46$, and 0.42 , respectively; as before, perfect prediction of responses to grating pairs by their component means would produce a slope of 0.5 . These data indicate that divisive suppression produced by superimposed gratings was essentially devoid of dynamics, and was present in close to its mature form from the earliest measurable response (Fig. 6e,f).

\section{Discussion}

We find that patterns of V1 population activity evoked by multiple simultaneous gratings differing in orientation were predicted with high precision by a simple form of divisive suppression. Specifically, activity patterns evoked by two superimposed gratings of identical contrast were well predicted by the mean of patterns evoked by each grating presented alone. This prediction was accurate for cortical neurons of all preferred orientations relative to the stimuli, and a wide range of orientation pairs. Moreover, the component mean was equally effective in predicting multiple grating responses for summed and non-summed grating pairs, and at both high and low contrasts. Single-unit recordings confirm that these population measures reflect the average activity of individual V1 neurons, that suppression is evident at the earliest stages of cortical activation, and that it continues to account for the population response throughout the duration of the stimulus period. These results indicate that divisive suppression and the response rescaling it produces are highly consistent features of the V1 population response to multiple orientations under a broad range of stimulus types. In the following sections we place these results in the context of previous studies, consider the implications of these findings for population coding mechanisms, and for understanding the mechanisms that mediate this fundamental property of cortical circuits.

The fact that superimposition of gratings of equivalent contrast yields a population response that is the average of the responses to the component gratings has not been noted in previous studies of cross-orientation suppression in V11-2, 3, 7, 24-27. In retrospect, this is understandable given the focus of most previous studies on how a non-optimal grating suppresses the response of neurons to their preferred grating stimulus, rather than how populations of neurons represent multiple grating stimuli. Indeed, previous studies have not typically reported the response evoked by the cross-oriented grating by itself, making it impossible to assess whether responses to superimposed gratings could have been predicted by the means of their component responses1, 3, 24, 25. Additionally, many studies have placed an emphasis on the effect of varying the contrast of the cross-oriented "mask" grating while keeping the contrast of the preferred grating fixed, and have shown that the strength of 
suppression varies with mask contrast. These results are not inconsistent with our own, in which responses to superimposed gratings are predicted by their component means only when components have equal contrast.

We note, however, that in those situations where responses to both component gratings have been reported, responses of V1 neurons to superimposed gratings of equivalent contrast often appear to fall close to the component average 7, 26, 27. Moreover, single-unit studies in extrastriate areas have shown that the mean of the component responses generally predicts activity evoked by multiple stimuli, such as colored bars in V2 and V4 31-33, directions of motion in areas MT and MST 34, 35, and shapes in IT 36. However, as our own single unit data indicate, even with component gratings that are identical except for their orientation, there is cell-to-cell variability in the strength of suppression, with some examples that exceed and others that fall short of the mean of the component responses. One average, responses to superimposed gratings are predicted by the component mean; whether functionally distinct subclasses of neurons exhibit characteristic and systematic departures from this prediction remains an open question.

What makes our results particularly intriguing is that they do not fit well with the traditional view of cross-orientation suppression as an outcome of a contrast gain control mechanism that prevents saturation from distorting the distributed representation of stimulus features 2 , 4-7. If saturation avoidance were the principal purpose of suppression, then we should find that the amount of suppression varies with the contrast of the component gratings. For instance, the superimposition of two low-contrast gratings requires little or no suppression to prevent the peak of the population response from reaching saturation. In spite of this, we find that the component mean predicts responses to equal-contrast superimposed gratings at both high and low contrasts (but see 26).

This consistency suggests that cross-orientation suppression is dictated by factors other than the potential for response saturation. We propose instead that cross-orientation suppression is the single-cell manifestation of a population coding scheme that maintains independent codes for stimulus orientation(s) and strength (e.g., contrast): the orientations present in a local (RF-sized) region of the stimulus are encoded by the relative activity of units tuned to different orientations (i.e., the shape of the population response), while stimulus strength is separately encoded by the summed activity of the entire local population. Specifically, cross-orientation suppression results from the redistribution of cortical activity necessary to represent the more complex orientation spectrum of the superimposed stimulus, without changing the total amount of cortical activity devoted to its representation (Supplementary Figure 4). When two gratings of the same contrast are superimposed, the level of population activity to which they are entitled by their contrast is distributed equally between them. One outcome of this redistribution is that the resulting profile of population activity is the mean of the profiles evoked by each when presented alone (when each grating is afforded the full measure of cortical activity commensurate with its contrast). A related outcome is that the responses of individual neurons preferring one of the gratings are smaller than when that grating is shown by itself in order to compensate for increased activity among neurons preferring the second grating, hence "cross-orientation suppression". Furthermore, what we observed as the "failure" of the predictive power of the component mean when two gratings 
differ in contrast simply reflects the fact that the redistribution of cortical activity is weighted by the relative contrast of each grating. This weighting is necessary to ensure that the distribution of activity across neurons with different preferred orientations accurately encodes the relative intensities of the gratings.

One notable feature of our results is the fact that the summed population response to a pair of superimposed gratings is identical to the summed population response evoked by one of the components presented by itself. In other words, superimposing an additional grating of the same contrast does not change the total amount of cortical activity representing the stimulus. Although this at first seems at odds with the fact that linear summation of two gratings will produce a new stimulus with twice the Michelson contrast of each component, it makes sense when one notes that that this increased contrast is totally invisible to any particular oriented filter. Thus, although the new stimulus is more elaborate in its orientation spectrum, its peak contrast viewed through a bank of filters is the same as for each of its component gratings.

Our results emphasize that the distributed coding of superimposed gratings is fundamentally a zero-sum operation. Adding elements of the same contrast to a stimulus reduces the amount of neural activity that is "available" under the contrast code to represent each of the components. We predict that addition of more gratings at the same contrast should only further broaden the distribution of population activity, without increasing the total amount of population activity representing the stimulus. (Consistent with this, we note that the component mean has been observed to predicted single-neuron responses to three stimuli in IT 36.) By the same token, changing the contrast of the stimulus without changing its orientation composition will change the total amount of cortical activity, without changing the relative distribution of the activity pattern.

Though the idea that the shape of the population response represents the stimulus orientation spectrum is fairly conventional, the idea that the total population activity, i.e. the area under the curve of the population response, could provide an independent representation of stimulus intensity has not figured prominently in previous discussions of population coding. However, a role for summed population activity as an independent measure of overall stimulus strength has been featured in at least one model of population-based probabilistic stimulus decoding 38. Consistent with this role, we find that plotting PRP area versus contrast of a single grating yields a contrast response function very similar to those of individual neurons (Fig. 7, also see 37). Moreover, the consistency of the component mean in predicting responses to superimposed gratings across contrasts indicates that a similar contrast response function exists for any pair of superimposed gratings, allowing summed population activity to signal the contrasts of more complex stimuli as well.

The applicability of our results to combinations of stimuli differing along dimensions other than orientation is an open question. It is important to emphasize that we do not propose that the component mean should predict responses to any combination of equalstrength stimuli. Rather, it should only apply when the combined stimulus requires that V1 encode multiple simultaneous values along the dimension in question. For instance, when two gratings differing in orientation are superimposed, their combined response resembles the component 
mean because the combined stimulus contains information about both orientations, evinced by the dual peaks in the stimulus' 2D frequency spectrum. On the other hand, the response to a stimulus derived from simple summation of two gratings that are identical in orientation will not be predicted by the component mean: the "compound" stimulus is simply a single grating at a higher contrast. As such, it has only a single frequency peak, and is "seen" by $\mathrm{V} 1$ as a single feature. This caveat does leave open the possibility that the component mean may predict responses to other compound stimuli that contain information about their source components, such as the sum of two gratings differing in spatial frequency. In any such case, however, the extent to which the compound stimulus will evoke the component mean will be critically dependent upon the components having equal strengths. Thus, while we might predict the same suppressive mechanisms to sculpt population activity for a stimulus that combines multiple spatial frequencies, the overall bandpass sensitivity of V1 in the frequency domain limits the range of frequency combinations under which we would expect it to produce the mean per se.

The mechanism underlying cross-orientation suppression has long been a subject of debate. Under the "normalization" model5, 7, cross-orientation suppression was proposed to be mediated by inhibitory feedback driven by the pooled activity of all orientationtuned units. Subsequent work led to the proposal that suppression was an outcome of activity-dependent depression of thalamocortical synapses, rather than cortical inhibition 39, 40. However, this explanation has been challenged by the observation that synaptic depression at the geniculocortical synapse is insufficient to explain the amount of suppression induced by a cross-oriented grating41. More recent work has demonstrated that cross-orientation suppression of layer 4 simple cells is accompanied by a decrease in both excitatory and inhibitory currents, effectively ruling out cortical inhibition as a source of suppression 26 . Two recent studies 26, 27 have proposed that cross-orientation suppression arises from nonlinearities in the responses of LGN neurons which are further amplified by the nonlinearities of spike threshold at the geniculo-cortical synapse.

The results presented here are difficult to reconcile with all of the proposed mechanisms of divisive suppression. Consistent with previous reports of fast cross-orientation suppression 25, the presence of suppression from the very onset of subthreshold responses seems to preclude involvement of inhibitory feedback. We observed suppression with stationary gratings, while the synaptic depression model predicts suppression exclusively for drifting gratings 40 . Similarly, models which attribute much of the suppression to LGN saturation predict little or no suppression for gratings that do not sum linearly - that is, those that do not produce regions of saturating contrast 26, 27. Against this, we observed the same strength of suppression for both non-summing rectangle-wave gratings and summed sinusoids. Additionally, these models predict that suppression should diminish at low contrast. Instead, we found that the strength of suppression was essentially identical for sinusoidal gratings at $25 \%$ and $12.5 \%$ contrast, even though the difference in activity evoked by single gratings at each contrast showed that cortical neurons - and, presumably, their thalamic inputs - were not near saturation in the low-contrast conditions.

The only previous study using intracellular recordings to examine $\mathrm{V} 1$ responses to multiple gratings found far greater cross-orientation suppression (roughly twice as much) in spike 
rate than in membrane potential, suggesting that a non-linear relationship between membrane potential and spike rate plays a significant role in amplifying suppressive effects 26 . In contrast, we found that the component mean predicted membrane potentials about as well as it predicted spike rates. Several factors may explain this difference. Previously, intracellular responses were characterized principally by measuring the magnitude of the modulation of spike rate and membrane potential at the fundamental frequency of a drifting stimulus, after discarding data from the first stimulus cycle 26 . On the other hand, we used stationary stimuli and characterized membrane responses as departures from resting potential averaged within discrete time windows following response onset, and spiking responses as average firing rate during the full $500 \mathrm{~ms}$ stimulus epoch. Another differentiating factor was the way in which we removed action potentials from membrane voltage records. Instead of using a $5 \mathrm{~ms}$ mean filter 26 , we truncated deloparizations past a manual estimate of spike threshold. Both methods likely distort the relationship between membrane potential and spike rate to a certain extent. Our use of a stationary stimulus, however, allowed us to look at extremely early time points in membrane responses, before spike threshold was reached. Notably, we observe that even at these earliest time points, membrane potentials for superimposed gratings were well predicted by the mean of the component responses. While the discrepancy between previous studies and our own may be attributable to laminar differences (i.e., cortical layer 4 versus layer 2/3), or species differences, we conclude that cross-orientation suppression in layer $2 / 3$ can be explained without the need to invoke the non-linearities of spike threshold.

One feature common to many proposed models of cross-orientation suppression is the assumption that it stems, either directly or indirectly, from the same mechanisms that produce contrast saturation 4, 5, 7, 26,27. Our results challenge this outlook: the ability of the component mean to predict responses at different contrasts suggests that crossorientation suppression should be viewed as part of a separate process. Moreover, viewed from the perspective of a population code that jointly represents the orientation spectrum and the effectiveness of a broad range of stimuli, the term `suppression' itself may be a misnomer. The orientation spectrum of the compound grating stimulus is fundamentally different from that of the single grating stimulus and, in this respect, the mechanism responsible for 'cross orientation suppression' may be similar to the reduction in response that occurs when an optimal grating stimulus is replaced with a less optimal grating, a reduction in response that few would regard as 'suppression'. Of course, recasting the phenomenon in this way does not reveal the circuitry that produces it. Further studies that probe the behavior of identified sub-populations of cortical neurons and their interactions will be necessary to gain insights into this fundamental behavior of cortical circuits.

\section{Methods}

Experiments were performed on juvenile tree shrews (Tupaia belangeri) of both sexes. All procedures conformed to National Institutes of Health guidelines, and were approved by the Duke University Animal Care and Use Committee. 


\section{Surgery and animal preparation}

Animal preparation has been described in detail previously42. Briefly, anesthesia was induced with a combination of ketamine and xylazine, and was maintained $2 \%$ halothane in a 1:1 mixture of $\mathrm{N} 2 \mathrm{O}$ and $\mathrm{O} 2$. After placement in a stereotactic device, animals were paralyzed and respirated to maintain end-tidal CO2 between 3-4\%. During imaging/ recording, halothane was reduced to $\sim 1 \%$ and adjusted as needed to maintain a stable heart rate. Temperature was maintained at $38^{\circ} \mathrm{C}$ with a thermal blanket.

For intrinsic signal imaging experiments, the scalp was incised and the bone over V1 was thinned with a scalpel blade. A dental wax chamber was mounted to the skull, filled with agar, and topped with a glass coverslip. Optical signals were imaged with an Imager 3001 (Optical Imaging Inc.) under 690nm illumination.

For extra- and intracellular electrode recordings, a small hole was made in the skull and the underlying dura was reflected. Extracellular recordings were made with glass-coated tungsten electrodes (FHC Inc.). Signals were band-pass filtered between 300 and $3000 \mathrm{~Hz}$, digitized at $10 \mathrm{kHz}$ and recorded for offline analysis. Intracellular recordings were made with sharp electrodes (90-130M $\Omega$ ) filled with $2 \mathrm{M} \mathrm{KAc}$. Potentials were recorded without filtering for offline analysis.

\section{Visual stimuli}

In imaging experiments, the principal experimental imaging stimulus consisted of stationary rectangle-wave gratings composed of $0.25^{\circ}$ wide lines, separated by $3^{\circ}$, presented against a gray background (insets, Figure 1A-C,F-H); this spacing was sufficiently close to ensure uniform stimulation of the cortex (Supplementary Figure 5). The $0.25^{\circ}$ lines alternated in unison between light and dark (relative to the fixed-luminance background) at $2 \mathrm{~Hz}$ in order to maintain cortical activity during image acquisition. During both the light and dark phases, Michelson contrast was 0.5 . When superimposed, gratings appeared to be "overlaid", as if one transparent sheet with thin lines were placed on another transparent sheet with lines of a different orientation, and the two laid together on a gray background. The luminance of pixels where the thin lines crossed was the same as for a single grating. A single experimental run generally contained four stimulus conditions: two single gratings of differing orientations, a superimposed condition with the same two orientations overlaid, plus a blank. Stimuli appeared for 4.5 seconds, with an 8 second interstimulus interval to allow intrinsic signals to decay to baseline (Supplementary Figure 6). Stimulus conditions were randomly interleaved and repeated 20-100 times.

In some experiments we used drifting achromatic sinusoidal gratings. Gratings were 0.25 cycles $/{ }^{\circ}$ and drifted at $4 \mathrm{~Hz}$. When presented as superimposed pairs, sinusoidal gratings combined linearly (i.e. Michelson contrast for superimposed gratings was twice that of each individual component).

In separate runs, we also acquired images during presentation of drifting square wave gratings $\left(0.4 \mathrm{cycles}^{\circ}, 4 \mathrm{~Hz}\right)$ at four orientations $\left(0^{\circ}, 45^{\circ}, 90^{\circ}\right.$, and $\left.135^{\circ}\right)$. We subsequently used these data to generate orientation preference maps according to a vector-sum method. 
Stimuli for both extra- and intracellular recordings were identical to stationary gratings used during optical imaging. Stimuli were presented for $500 \mathrm{~ms}$ with a 2 second ISI. Only the light phase of each stimulus was used (i.e., light bars on the gray background). The standard stimulus set consisted of single gratings at 8 different orientations and 28 conditions consisting of every combination of the 8 base orientations. Stimuli were randomly interleaved, and responses were averaged over 15-40 repetitions.

\section{Data acquisition and analysis}

We averaged images of the cortex within $500 \mathrm{~ms}$ frames beginning $500 \mathrm{~ms}$ before stimulus onset. After first-frame subtraction, images were converted to percent-reflectance-change maps $(\Delta R / R)$ by dividing them by the same first frame. Each $\Delta R / R$ map was then high-pass filtered by subtracting a $1 \mathrm{~mm}$ mean-filtered version of the same map, and then passed through a $0.05 \mathrm{~mm}$ mean filter to remove high frequencies.

To relate population responses evoked by superimposed gratings to activity evoked by single gratings, we derived Population Response Profiles (PRPs) for each stimulus condition 14 . We used orientation preference maps to assign a preferred orientation tag to each pixel, ranging from $0^{\circ}$ to $179^{\circ}$ in $0.7^{\circ}$ steps. Next, for each image acquired during presentation of an experimental stimulus (e.g., a pair of superimposed gratings), we computed the average signal intensity among all pixels with the same orientation tag. We rebinned these averages at $10^{\circ}$ increments and plotted them as a function of orientation tag to produce PRPs. Because the high-pass filtering of the input images amounted to a meanvalue subtraction, the resulting PRPs no longer represented $\Delta R / R$, and frequently contained negative numbers. To avoid confusion, we assembled PRPs into families composed of one PRP for a superimposed pair and one for each of its component gratings, and defined the most negative value among all points in each family as zero. We then set the maximum value in the family to 1 , and scaled all other values accordingly. It is critical to note that neither of these last two steps in any way changes the relative values of PRPs within a stimulus family.

During extracellular recordings, we performed preliminary online spike sorting using Spike2 software (CED). Once we isolated the response of a single neuron, we employed automated procedures to determine RF position and preferred orientation. Only cells from which we recorded at least 15 repetitions of each of our experimental stimuli were included in further analysis. We adjusted the position of gratings for each neuron to ensure that one crossingpoint of each superimposed pairs was located as close as possible to the center of its RF. (This applied to intracellular recordings as well.) More precise offline spike sorting frequently permitted us to extract the response of more than one neuron from each stimulus run.

During intracellular recordings, we only analyzed data from cells with resting potentials below $-60 \mathrm{mV}$ and action potential peaks exceeding $0 \mathrm{mV}$. To analyze the evolution of responses over time, we computed response latency for each superimposed grating pair by identifying the earliest $0.1 \mathrm{~ms}$ bin in the response to the more-preferred component that had a voltage above rest with a probability of less than 0.01 , based upon the distribution of voltages recorded during the first $20 \mathrm{~ms}$ following stimulus onset. This criterion 
corresponded to a median depolarization of $1.8 \mathrm{mV}$ from rest, and identified response onsets with a median latency of $50.7 \mathrm{~ms}$, both averaged across all grating pairs from all neurons. This relatively long median latency reflects the impact grating pairs which evoked weak and slowly-rising responses.

\section{Supplementary Material}

Refer to Web version on PubMed Central for supplementary material.

\section{Acknowledgments}

The authors wish to thank the members of the Fitzpatrick lab for helpful discussions and assistance with data collection and analysis. Current address for S. MacEvoy: Center for Cognitive Neuroscience, University of Pennsylvania, Philadelphia, PA 19104. Current address for T. Tucker: Department of Biology, Guilford College, Greensboro, NC 27410. This work was supported by National Institutes of Health grants EY06821 to D. Fitzpatrick and EY016319 to S. MacEvoy.

\section{References}

1. Morrone MC, Burr DC, Maffei L. Functional implications of cross-orientation inhibition of cortical visual cells. I. Neurophysiological evidence. Proc R Soc Lond B Biol Sci. 1982; 216:335-354. [PubMed: 6129633]

2. Bonds AB. Role of inhibition in the specification of orientation selectivity of cells in the cat striate cortex. Vis Neurosci. 1989; 2:41-55. [PubMed: 2487637]

3. DeAngelis GC, Robson JG, Ohzawa I, Freeman RD. Organization of suppression in receptive fields of neurons in cat visual cortex. J Neurophysiol. 1992; 68:144-163. [PubMed: 1517820]

4. Geisler WS, Albrecht DG. Cortical neurons: isolation of contrast gain control. Vision Res. 1992; 32:1409-1410. [PubMed: 1455713]

5. Heeger DJ. Normalization of cell responses in cat striate cortex. Vis Neurosci. 1992; 9:181-197. [PubMed: 1504027]

6. Carandini M, Heeger DJ. Summation and division by neurons in primate visual cortex. Science. 1994; 264:1333-1336. [PubMed: 8191289]

7. Carandini M, Heeger DJ, Movshon JA. Linearity and normalization in simple cells of the macaque primary visual cortex. J Neurosci. 1997; 17:8621-8644. [PubMed: 9334433]

8. Simoncelli, EP.; Schwartz, O. Image statistics and cortical normalization models. In: Kearns, MS.; Sollar, SA.; Cohn, DA., editors. Advances in Neural Information Processing Systems. MIT Press; Cambridge, MA: 1999. p. 153-159.

9. Valerio R, Navarro R. Optimal coding through divisive normalization models of V1 neurons. Network. 2003; 14:579-593. [PubMed: 12938772]

10. Schwartz O, Simoncelli EP. Natural signal statistics and sensory gain control. Nat Neurosci. 2001; 4:819-825. [PubMed: 11477428]

11. De Valois KK, De Valois RL, Yund EW. Responses of striate cortex cells to grating and checkerboard patterns. J Physiol. 1979; 291:483-505. [PubMed: 113531]

12. Pollen D, Ronner S. Visual cortical neurons as localized spatial-frequency filters. IEEE Transactions on Systems, Man, and Cybernetics. 1983; 13:907-916.

13. Adelson EH, Bergen JR. Spatiotemporal energy models for the perception of motion. J Opt Soc Am A. 1985; 2:284-299. [PubMed: 3973762]

14. Basole A, White LE, Fitzpatrick D. Mapping multiple features in the population response of visual cortex. Nature. 2003; 424:986-990. [PubMed: 12827202]

15. Basole A, Kreft-Kerekes V, White LE, Fitzpatrick D. Cortical cartography revisited: A frequency perspective on the functional architecture of visual cortex. Prog Brain Res. 2006; 154:121-134. [PubMed: 17010706] 
16. Skottun BC, Bradley A, Sclar G, Ohzawa I, Freeman RD. The effects of contrast on visual orientation and spatial frequency discrimination: a comparison of single cells and behavior. $\mathrm{J}$ Neurophysiol. 1987; 57:773-786. [PubMed: 3559701]

17. Sclar G, Freeman RD. Orientation selectivity in the cat's striate cortex is invariant with stimulus contrast. Exp Brain Res. 1982; 46:457-461. [PubMed: 7095050]

18. Maffei L, Fiorentini A. The unresponsive regions of visual cortical receptive fields. Vision Res. 1976; 16:1131-1139. [PubMed: 969225]

19. Knierim JJ, van Essen DC. Neuronal responses to static texture patterns in area V1 of the alert macaque monkey. J Neurophysiol. 1992; 67:961-980. [PubMed: 1588394]

20. Kapadia MK, Ito M, Gilbert CD, Westheimer G. Improvement in visual sensitivity by changes in local context: parallel studies in human observers and in V1 of alert monkeys. Neuron. 1995; 15:843-856. [PubMed: 7576633]

21. Sillito AM, Grieve KL, Jones HE, Cudeiro J, Davis J. Visual cortical mechanisms detecting focal orientation discontinuities. Nature. 1995; 378:492-496. [PubMed: 7477405]

22. Sengpiel F, Sen A, Blakemore C. Characteristics of surround inhibition in cat area 17. Exp Brain Res. 1997; 116:216-228. [PubMed: 9348122]

23. Kabara JF, Bonds AB. Modification of response functions of cat visual cortical cells by spatially congruent perturbing stimuli. J Neurophysiol. 2001; 86:2703-2714. [PubMed: 11731530]

24. Sengpiel F, Vorobyov V. Intracortical origins of interocular suppression in the visual cortex. J Neurosci. 2005; 25:6394-6400. [PubMed: 16000630]

25. Smith MA, Bair W, Movshon JA. Dynamics of suppression in macaque primary visual cortex. J Neurosci. 2006; 26:4826-4834. [PubMed: 16672656]

26. Priebe NJ, Ferster D. Mechanisms underlying cross-orientation suppression in cat visual cortex. Nat Neurosci. 2006; 9:552-561. [PubMed: 16520737]

27. Li B, Thompson JK, Duong T, Peterson MR, Freeman RD. Origins of cross-orientation suppression in the visual cortex. J Neurophysiol. 2006; 96:1755-1764. [PubMed: 16855109]

28. Rust NC, Movshon JA. In praise of artifice. Nat Neurosci. 2005; 8:1647-1650. [PubMed: 16306892]

29. Bosking WH, Kretz R, Pucak ML, Fitzpatrick D. Functional specificity of callosal connections in tree shrew striate cortex. J Neurosci. 2000; 20:2346-2359. [PubMed: 10704509]

30. Chisum HJ, Mooser F, Fitzpatrick D. Emergent properties of layer 2/3 neurons reflect the collinear arrangement of horizontal connections in tree shrew visual cortex. J Neurosci. 2003; 23:29472960. [PubMed: 12684482]

31. Moran J, Desimone R. Selective attention gates visual processing in the extrastriate cortex. Science. 1985; 229:782-784. [PubMed: 4023713]

32. Luck SJ, Chelazzi L, Hillyard SA, Desimone R. Neural mechanisms of spatial selective attention in areas V1, V2, and V4 of macaque visual cortex. J Neurophysiol. 1997; 77:24-42. [PubMed: 9120566]

33. Reynolds JH, Chelazzi L, Desimone R. Competitive mechanisms subserve attention in macaque areas V2 and V4. J Neurosci. 1999; 19:1736-1753. [PubMed: 10024360]

34. Recanzone GH, Wurtz RH, Schwarz U. Responses of MT and MST neurons to one and two moving objects in the receptive field. J Neurophysiol. 1997; 78:2904-2915. [PubMed: 9405511]

35. Britten KH, Heuer HW. Spatial summation in the receptive fields of MT neurons. J Neurosci. 1999; 19:5074-5084. [PubMed: 10366640]

36. Zoccolan D, Cox DD, DiCarlo JJ. Multiple object response normalization in monkey inferotemporal cortex. Journal of Neuroscience. 2005; 25:8150-8164. [PubMed: 16148223]

37. Carandini M, Sengpiel F. Contrast invariance of functional maps in cat primary visual cortex. J Vis. 2004; 4:130-143. [PubMed: 15086304]

38. Zemel RS, Dayan P, Pouget A. Probabilistic interpretation of population codes. Neural Comput. 1998; 10:403-430. [PubMed: 9472488]

39. Freeman TC, Durand S, Kiper DC, Carandini M. Suppression without inhibition in visual cortex. Neuron. 2002; 35:759-771. [PubMed: 12194874] 
40. Carandini M, Heeger DJ, Senn W. A synaptic explanation of suppression in visual cortex. J Neurosci. 2002; 22:10053-10065. [PubMed: 12427863]

41. Boudreau CE, Ferster D. Short-term depression in thalamocortical synapses of cat primary visual cortex. J Neurosci. 2005; 25:7179-7190. [PubMed: 16079400]

42. Bosking WH, Zhang Y, Schofield B, Fitzpatrick D. Orientation selectivity and the arrangement of horizontal connections in tree shrew striate cortex. J Neurosci. 1997; 17:2112-2127. [PubMed: 9045738] 

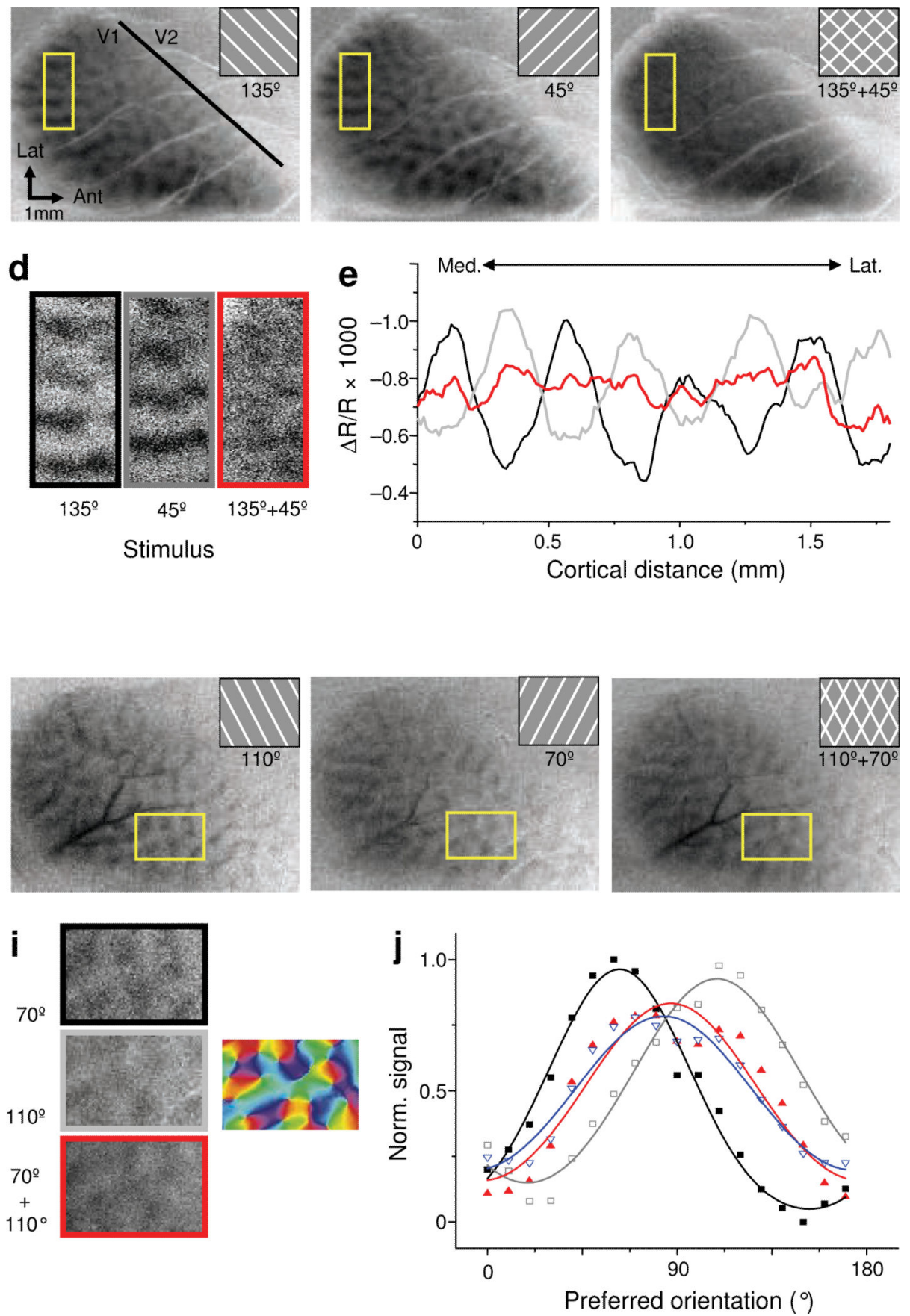

Figure 1.

Intrinsic signal optical images of tree shrew V1 acquired during presentation of single and superimposed gratings. (a-c) Single condition patterns evoked in one animal by stationary flashing rectangle-wave gratings identified by the icon in each panel. (d) High magnification views of ROIs denoted by yellow boxes in a-c. (e) Average optical signal within each row of pixels in the corresponding color-coded ROI in $\mathbf{d}$. Zero on the $\mathrm{x}$-axis corresponds to the bottom row of pixels. (f-h) Single condition images acquired from a different animal with gratings at $70^{\circ}$ and $110^{\circ}$. (i) Patterns of activation in each image were quantified by 
computing average optical signal values within blood vessel-free ROIs for all pixels with the same preferred orientation. Pixel orientation preferences were drawn from an orientation preference map derived from a separate data set. (j) Each PRP data point in $J$ represents the distribution of cortical activity evoked by single stimulus, follwing the color code in $\mathbf{i}$. A PRP generated from the average of the component grating ROI images is shown in blue. Curves are best-fit circular Gaussians. 

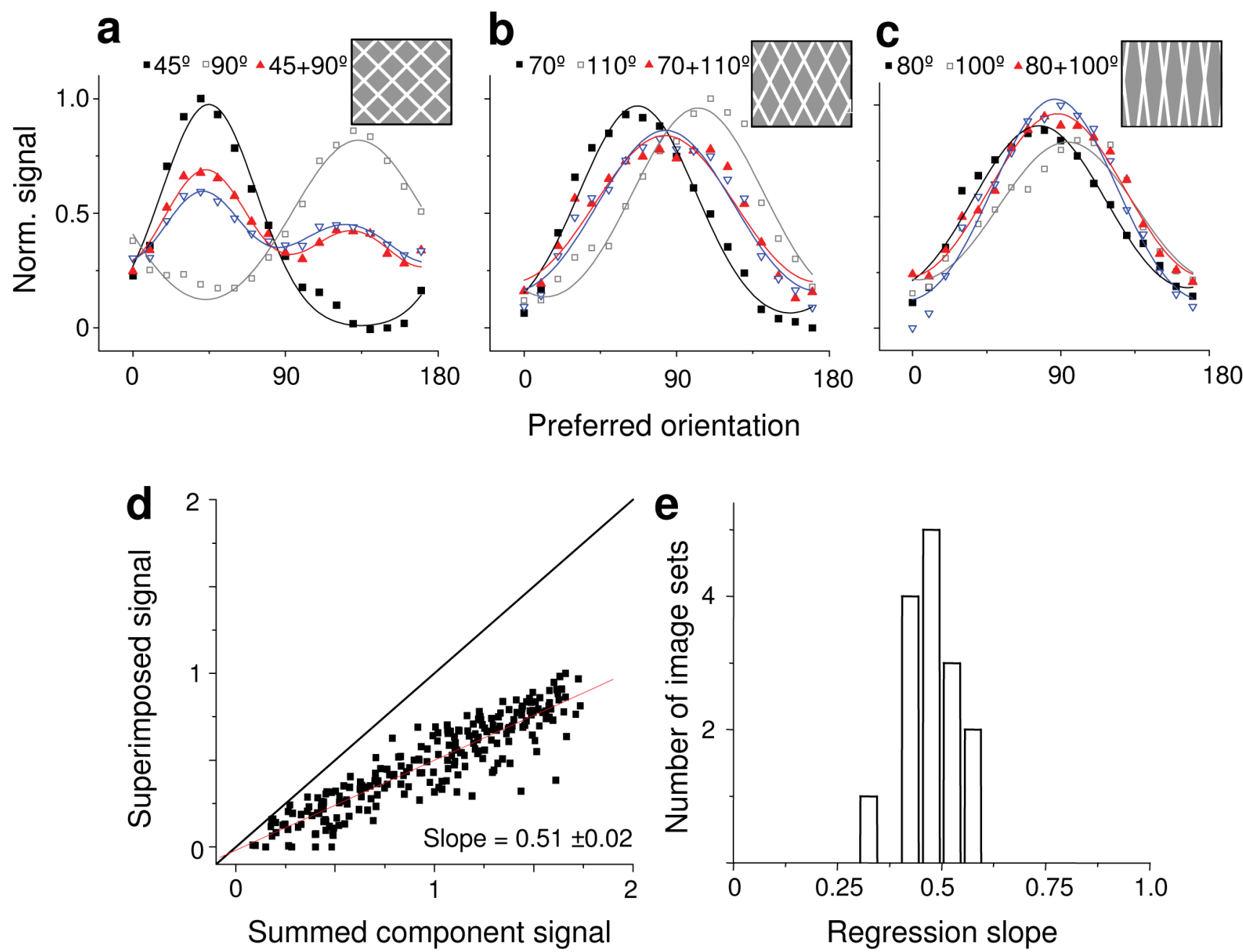

Figure 2.

The component mean consistently predicted responses to superimposed gratings. (a-c), Single animal PRPs for stimuli indicated by the color coded labels. PRPs for the mean of single grating images is shown in blue. Note that the predictor PRP was not defined simply as the average of the component PRPs, but was generated from the average cortical image evoked by the component gratings. Blue PRPs are derived from the average of single component images, not the average of single component PRPs; because imaging averaging took place prior to all other image processing steps, blue PRPs occasionally differ slightly from the average of single grating PRPs. (d) PRP data collapsed across 15 image sets from 8 animals, representing a range of orientation offsets. Each datum plots the activity evoked by a pairs of superimposed gratings evoked in one PRP bin with respect to the sum of activity evoked by the corresponding single gratings. Linear regression through these data produces a line with a slope of $0.51 . \mathrm{R}^{2}$ is 0.83 . (e) Distribution of regression slopes for individual PRP sets. Linear regressions were performed separately on each of the PRP sets contributing to d. The median slope value across PRP sets was 0.48 . 

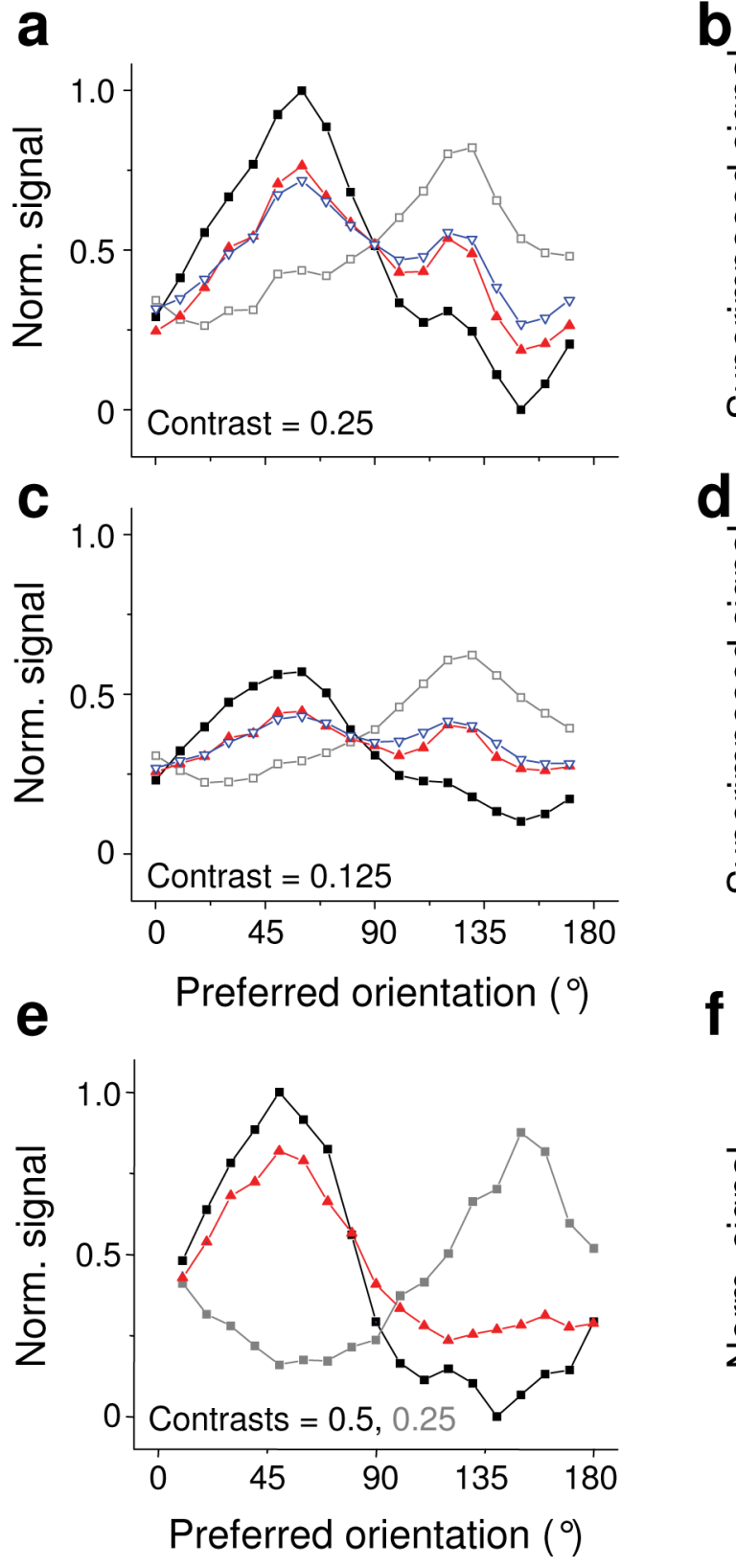

b
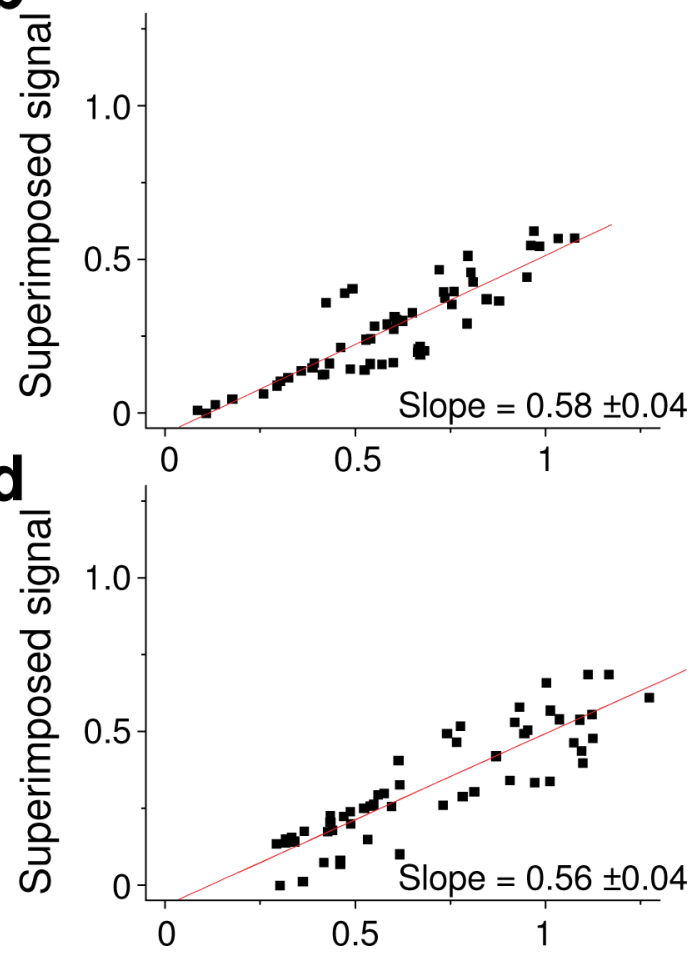

Summed comp. signal

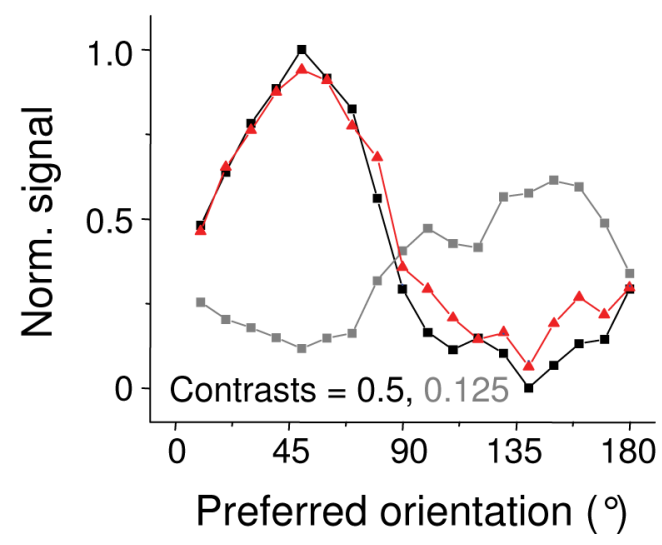

Figure 3.

Responses to superimposed sinusoidal gratings at different contrasts. (a,c) PRPs from one animal evoked by drifting sinusoidal gratings at $45^{\circ}$ and $135^{\circ}$ presented singly and as summed pairs, color coded as in Fig. 2. Contrasts of both gratings were $25 \%$ in a and $12.5 \%$ in c. Data in c were scaled according to maximum and minimum values in $\mathbf{a}$. (b,d) Combined PRP data from three animals for contrast of $25 \%$ and $12.5 \%$, respectively. (e,f) Single-animal PRPs for dissimilar component contrasts. 

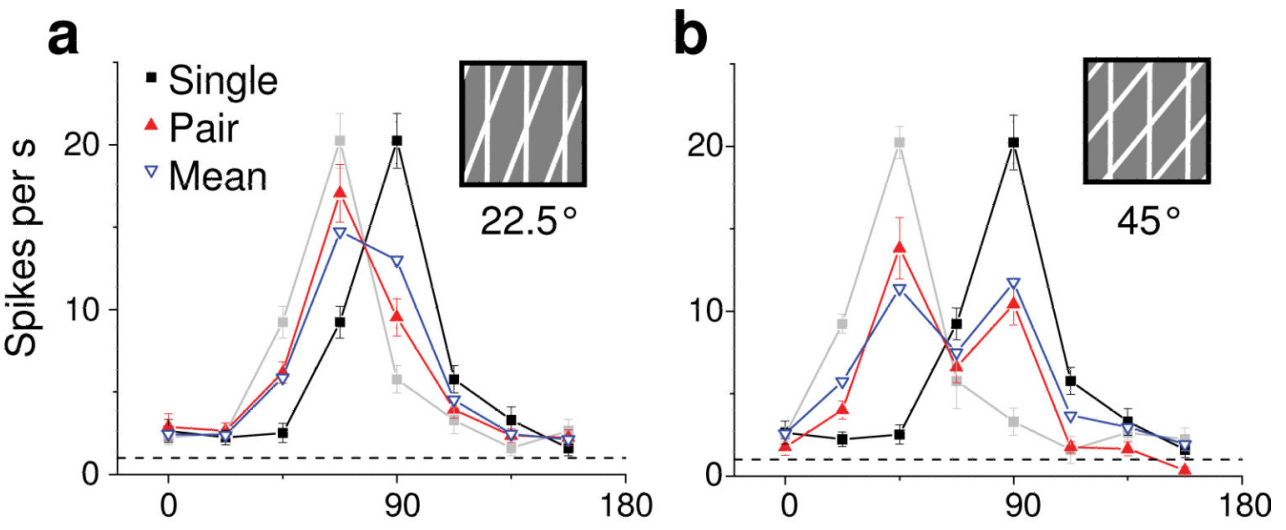

Base orientation ()
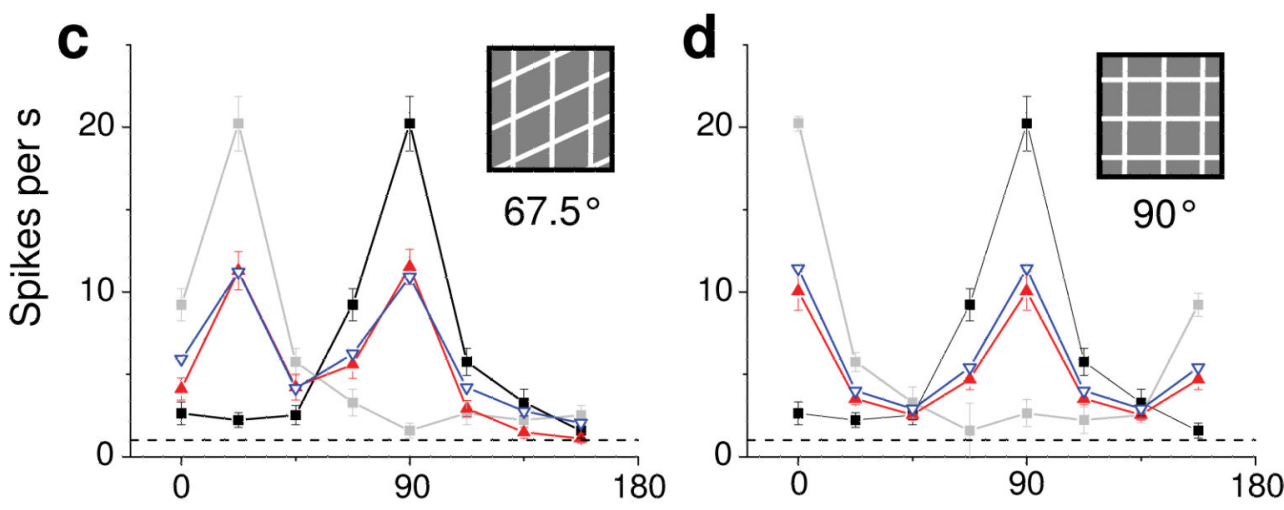

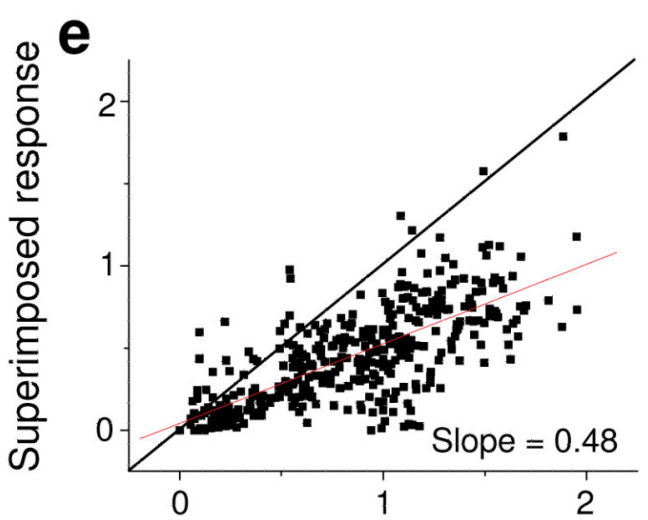

Summed comp. response

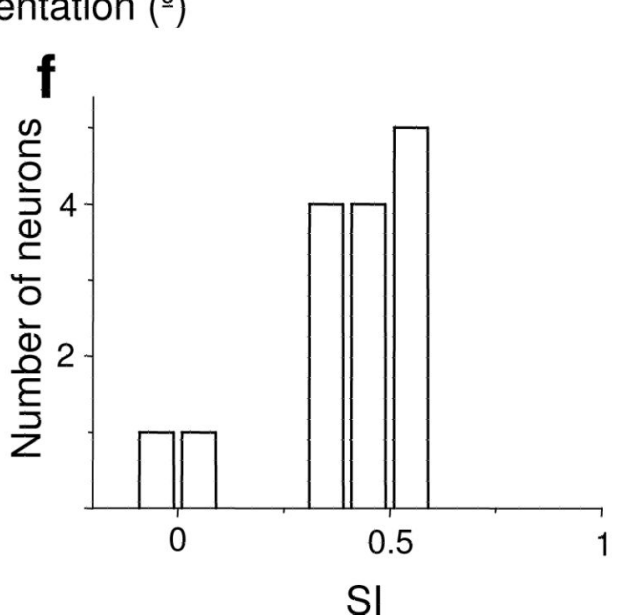

Figure 4.

Single-unit responses to single and superimposed gratings. (a-d) Tuning functions of one V1 neuron for single stationary gratings and fixed-offset grating pairs. Gray lines represent the single-grating tuning curve shifted by the angular offset indicated under the icon in each panel. Responses to pairs are plotted with respect to the orientation of a base grating, to which a second grating advanced in orientation by the offset value for that panel was added. Dashed line is spontaneous firing rate. Error bars are s.e.m. (e) Relationship between responses to superimposed gratings and summed component responses for all grating pairs 
across 15 neurons. Each datum represents the response of one neuron to a single grating pair (y-axis) plotted with respect to the sum of the responses to individual component gratings. Units are spikes per second normalized individually for each neuron to the most robust response across all stimuli. A regression line through these points has a slope of 0.48 ; perfect prediction by component means would result in a slope of $0.5 . \mathrm{R}^{2}$ is 0.55 . (f) Distribution of suppression indices (SI) across all neurons. The majority of SI values fell in a single cluster near 0.5; overall mean was 0.4. The cell illustrated in a-d had an SI of 0.58. 

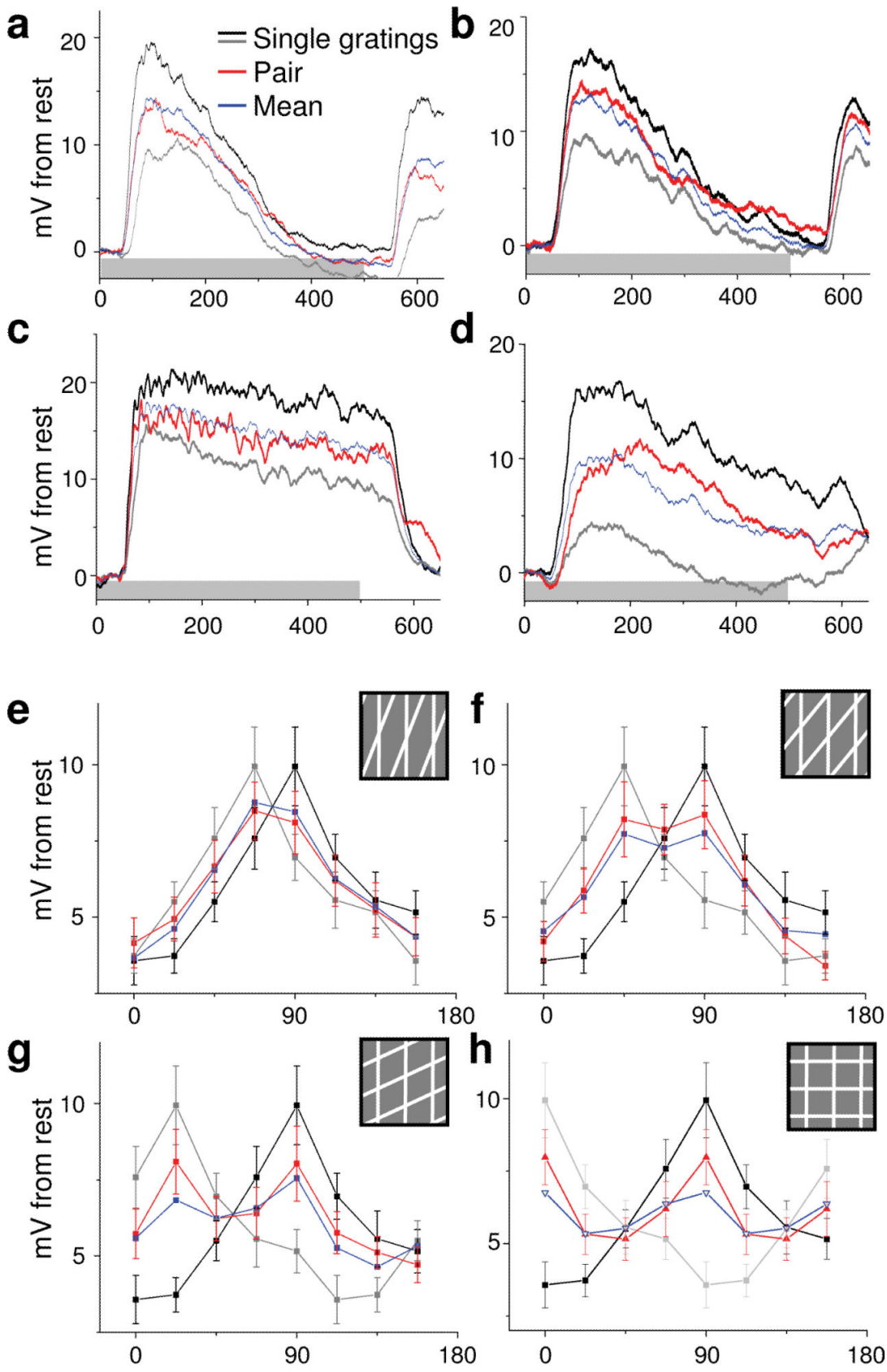

Base orientation ( $(\stackrel{)}{)}$

Figure 5.

Intracellularly recorded responses to single and superimposed gratings. (a-d) Responses of four neurons to select grating pairs. Angle between gratings was $67.5^{\circ}$ in $\mathbf{a}, 45^{\circ}$ in $\mathbf{b}, 22.5^{\circ}$ in $\mathbf{c}$, and $90^{\circ}$ in d. Depolarizations following stimulus offset are OFF responses. Shaded regions represents stimulus duration (e-h) Population average tuning functions for single and superimposed gratings ( $n=15$ neurons). Colors follow same convention as in a-d. Average membrane voltages during $400 \mathrm{~ms}$ poststimulus were averaged across all cells after aligning each neuron's preferred orientation to $90^{\circ}$. Error bars are s.e.m. 

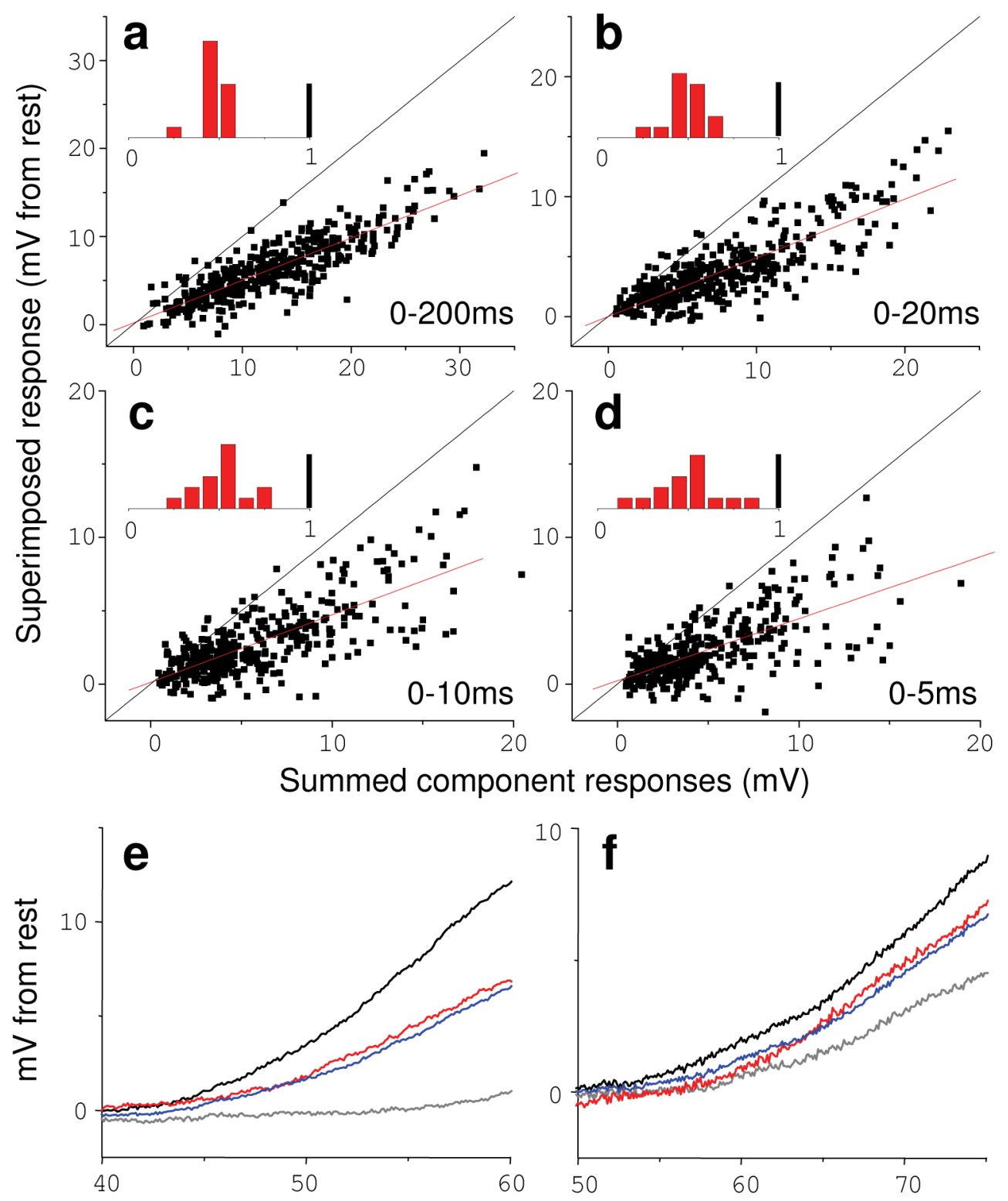

Time from stimulus onset (ms)

Figure 6.

Relationship of intracellularly recorded responses to superimposed and component gratings across time. (a-d) Main axes show combined responses of 15 neurons to superimposed gratings and summed responses to components, averaged over the time following response onset indicated in each panel. Regression slopes were $0.48,0.49,0.46$, and 0.42 respectively, and $\mathrm{R}^{2}$ values were $0.72,0.69,0.56$, and 0.46 . Insets show distribution of SI values across neurons for each time window; scale bar at 1 represents 5 neurons. SI values were calculated as described for extracellular recordings, except using average membrane potential within each time window. (e,f) Time-expanded view of responses of two neurons shown in $\mathbf{5 a , b}$, using the same color code. 


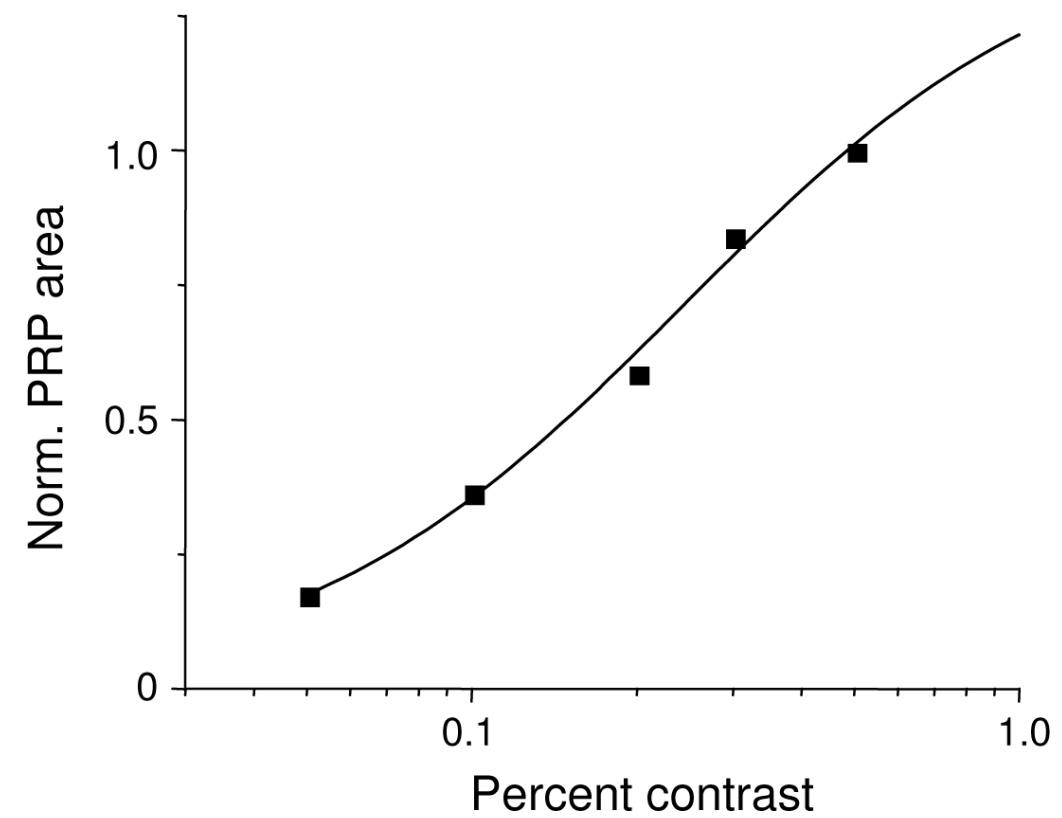

Figure 7.

Contrast response function derived from a single imaging session in one animal. Each point represents the area under the PRP for a single grating presented at the contrast shown. The area of each PRP was computed by first subtracting the minimum value from each point in that PRP, and then evaluating the integral of a circular Gaussian function fit to the results. Areas were normalized to the maximum across all contrasts. Data are fit with a NakaRushton function, with parameters $c_{50}=0.24 \pm 0.13$ and $n=1.24 \pm 0.35$; these compare to values of 0.33 and 1.64 , respectively, found with optical imaging in cat V137. 